\title{
Regularity of a complex Monge-Ampère equation on Hermitian manifolds
}

\author{
XiaOlan Nie
}

\begin{abstract}
We obtain higher-order estimates for a parabolic flow on a compact Hermitian manifold. As an application, we prove that a bounded $\hat{\omega}$-plurisubharmonic solution of an elliptic complex Monge-Ampère equation is smooth under an assumption on the background Hermitian metric $\hat{\omega}$. This generalizes a result of Székelyhidi and Tosatti on Kähler manifolds.
\end{abstract}

\section{Introduction}

In [18], Székelyhidi and Tosatti studied regularity of weak solutions of the equation

$$
(\omega+\sqrt{-1} \partial \bar{\partial} \phi)^{n}=\mathrm{e}^{-F(\phi, z)} \omega^{n}
$$

on an $n$-dimensional compact Kähler manifold $(M, \omega)$, where $F: \mathbb{R} \times M \rightarrow$ $\mathbb{R}$ is a smooth function and $\omega+\sqrt{-1} \partial \bar{\partial} \phi \geq 0$ in the sense of currents.

According to the local theory of Bedford-Taylor [1], for a locally bounded plurisubharmonic function $u$, the wedge products $\left(d d^{c} u\right)^{k}, 1 \leq k \leq n$ are well defined, where $d^{c}=\frac{\sqrt{-1}}{2}(\bar{\partial}-\partial)$ and $d d^{c}=\sqrt{-1} \partial \bar{\partial}$. Indeed, for such $u$ and $T$ a positive closed current, the current $u T$ is well defined and

$$
d d^{c} u \wedge T:=d d^{c}(u T)
$$

is also a positive closed current. Then the wedge products $\left(d d^{c} u\right)^{k}, 1 \leq k \leq n$ can be defined inductively as closed positive currents. Denote

$$
\begin{gathered}
\operatorname{PSH}(M, \omega)=\{u: M \rightarrow[-\infty,+\infty) \mid u \text { is upper semicontinuous, } \\
\omega+\sqrt{-1} \partial \bar{\partial} u \geq 0\}
\end{gathered}
$$

the set of $\omega$-plurisubharmonic (short for $\omega$-psh) functions on $M$. If $\phi \in$ $P S H(M, \omega) \cap L^{\infty}(M)$ solves Equation (1.1) in the above sense of BedfordTaylor, we say $\phi$ is a weak solution of the equation. The Hölder continuity 
of weak solutions follows from Kołodziej [12]. Using a different approach, Székelyhidi and Tosatti [18] proved that such weak solutions are actually smooth. Particularly, if $M$ is Fano, $\omega \in c_{1}(M)$ and $F(\phi, z)=\phi-h$, where $h$ satisfies $\sqrt{-1} \partial \bar{\partial} h=\operatorname{Ric}(\omega)-\omega$, their result implies that Kähler-Einstein currents with bounded potentials are smooth.

In the proof of [18], the authors use the smoothing property of the corresponding parabolic flow:

$$
\frac{\partial \varphi}{\partial t}=\log \frac{(\omega+\sqrt{-1} \partial \bar{\partial} \varphi)^{n}}{\omega^{n}}+F(\varphi, z) .
$$

They construct a function $\varphi \in C^{0}([0, T] \times M) \cap C^{\infty}((0, T] \times M)$ with $\varphi(0)=$ $\phi$ which solves Equation (1.2) on $(0, T]$, where $T$ depends only on $\sup |\phi|, F$ and $\omega$. Then they show that $\dot{\varphi}(t)=0$ for $0<t \leq T$, since the initial $\phi$ is a solution of (1.1). Therefore, $\phi=\varphi(0)=\varphi(t)$ is smooth. Similar construction was previously used in Song-Tian [17] for the Kähler-Ricci flow.

As Equation (1.1) also makes sense on Hermitian manifolds, it is natural to consider the regularity of weak solutions of Equation (1.1) in a more general setting. On a Hermitian manifold, there are no local potentials for $\omega$. However, $\omega$-psh functions are locally the sum of plurisubharmonic functions and smooth functions. Using that the wedge product of a smooth positive $(1,1)$ form and a positive current is again a positive current, the current $(\omega+\sqrt{-1} \partial \bar{\partial} \phi)^{n}$ is still well defined for bounded $\omega$-psh functions. For more details on pluripotential theory, we refer to $[1,3,8-10]$.

In this note, we show that the higher-order estimates in [18] can be obtained on compact Hermitian manifolds. Particularly, the flow (1.2) with smooth initial data $\varphi_{0}$ has a smooth solution for a time $T$ which depends only on $\sup \left|\varphi_{0}\right|, \sup \left|\dot{\varphi}_{0}\right|$. Then we obtain the following theorem.

Theorem 1.1. Let $(M, \hat{g})$ be an $n$-dimensional compact Hermitian manifold with the fundamental 2-form $\hat{\omega}$ satisfying

$$
\forall u \in P S H(M, \hat{\omega}) \cap L^{\infty}(M), \quad \int_{M}(\hat{\omega}+\sqrt{-1} \partial \bar{\partial} u)^{n}=\int_{M} \hat{\omega}^{n}
$$

and $F: \mathbb{R} \times M \rightarrow \mathbb{R}$ be a smooth function. Suppose that $\phi \in P S H(M, \hat{\omega}) \cap$ $L^{\infty}(M)$ solves

$$
(\hat{\omega}+\sqrt{-1} \partial \bar{\partial} \phi)^{n}=\mathrm{e}^{-F(\phi, z)} \hat{\omega}^{n}
$$

in the sense of currents. Then $\phi$ is smooth. 
Here the assumption (1.3) is automatically true on Kähler manifolds. In [18], the proof of the above theorem on Kähler manifolds needs Kołodziej's stability result [11]. We use the assumption (1.3) from [3], under which the usual comparison principle is true, to make sure the stability result holds on such Hermitian manifolds [13]. Particularly, if $\hat{\omega}$ satisfies GuanLi's [6] condition $\partial \bar{\partial} \hat{\omega}^{k}=0, k=1,2$, the assumption is satisfied. When $M$ is a complex surface, such metrics always exist due to a result of Gauduchon [4].

In the proof of our theorem, the main difference between the Hermitian case and Kählar case lies in the $C^{2}, C^{3}$ estimates and bound for $|\mathrm{Ric}|$. The computation on Hermitian manifolds is more complicated due to the existence of torsion terms. The proof of the second-order estimate follows closely the arguments of Gill [5] and Tosatti-Weinkove [20]. For the thirdorder estimate, we make use of the arguments in Phong-Sešum-Sturm [14] and Sherman-Weinkove [19]. Such estimate for the first derivative of the evolving Hermitian metrics was also established in [24], where the authors took a local reference Kähler metric to obtain a good bound. To bound $\mid$ Ric $\mid$, we need to deal with the new terms involving $\mid \nabla$ Ric $\mid$ very carefully.

The techniques used in this paper can be applied to construct a weak solution of the Chern-Ricci flow [22-24] with singular initial Gauduchon metric on complex surfaces. In [22], Tosatti and Weinkove conjectured that if the Chern-Ricci flow starting from a Gauduchon metric is non-collapsing in finite time, then it blows down finitely many exceptional curves and continues in a unique way on a new complex surface. They proved in [23] the smooth convergence of the metrics away from the exceptional curves and the global Gromov-Hausdorff convergence (under a suitable condition) as $t$ approaches the singular time. It is expected that the flow can continue on the new surface from the push-down of the limiting current. We will investigate this in further work.

The paper is organized as follows. In Section 2, we give some background material on Hermitian manifolds. Then we use maximum principle to obtain the estimates for existence of the parabolic flow for a short time depending only on $\sup \left|\varphi_{0}\right|, \sup \left|\dot{\varphi}_{0}\right|$. In Section 4 , we use the smoothing property of the parabolic flow to prove Theorem 1.1.

\section{Preliminaries}

For reader's convenience, in this section we introduce some basic material on Hermitian manifolds. The formulas given here can be found in [19].

Let $(M, g)$ be an $n$-dimensonal compact Hermitian manifold with the fundamental 2-form $\omega=\sqrt{-1} g_{i \bar{j}} d z^{i} \wedge d \bar{z}^{j}$ in local coordinates. Denote $\nabla$ 
the Chern connection of $g$ with Christoffel symbols $\Gamma_{i j}^{k}$ and torsion $T$ given by

$$
\Gamma_{i j}^{k}=g^{k \bar{l}} \partial_{i} g_{j \bar{l}}, \quad T_{i j}^{k}=\Gamma_{i j}^{k}-\Gamma_{j i}^{k}
$$

The covariant derivatives of $X=X^{j} \frac{\partial}{\partial z^{j}}$ and $a=a_{j} d z^{j}$ are defined in components as

$$
\nabla_{i} X^{j}=\partial_{i} X^{j}+\Gamma_{i k}^{j} X^{k}, \quad \nabla_{i} a_{j}=\partial_{i} a_{j}-\Gamma_{i j}^{k} a_{k}
$$

Then $\nabla$ can be extended naturally to any tensors. Define the Chern curvature tensor of $g$ in components to be

$$
R_{i \overline{j k}}^{l}=-\partial_{\bar{j}} \Gamma_{i k}^{l}
$$

We lower and raise indices using metric $g$. Then

$$
R_{i \bar{j} k \bar{l}}=-\partial_{i} \partial_{\bar{j}} g_{k \bar{l}}+g^{p \bar{q}} \partial_{i} g_{k \bar{q}} \partial_{\bar{j}} g_{p \bar{l}}
$$

and the Chern-Ricci tensor is given by

$$
R_{i \bar{j}}=g^{k \bar{l}} R_{i \bar{j} k \bar{l}}=-\partial_{i} \partial_{\bar{j}} \log \operatorname{det} g .
$$

We have the following commutation formulas:

$$
\begin{gathered}
{\left[\nabla_{i}, \nabla_{\bar{j}}\right] X^{l}=R_{i \bar{j} k}{ }^{l} X^{k}, \quad\left[\nabla_{i}, \nabla_{\bar{j}}\right] a_{k}=-R_{i \bar{j} k}{ }^{l} a_{l},} \\
{\left[\nabla_{i}, \nabla_{\bar{j}}\right] \overline{X^{l}}=-R_{i \bar{j} \bar{k}} \bar{X}^{k}, \quad\left[\nabla_{i}, \nabla_{\bar{j}}\right] \overline{a_{k}}=R_{i \bar{j} \bar{k}} \overline{a_{l}} .}
\end{gathered}
$$

The Bianchi identities will not hold necessarily for general Hermitian manifolds. There are extra torsion terms in the following identities:

$$
\begin{aligned}
R_{i \bar{j} k \bar{l}}-R_{k \bar{j} i \bar{l}} & =-\nabla_{\bar{j}} T_{i k \bar{l}}, \\
R_{i \bar{j} k \bar{l}}-R_{i \bar{l} k \bar{j}} & =-\nabla_{i} T_{\bar{j} \bar{l} k}, \\
R_{i \bar{j} k \bar{l}}-R_{k \bar{l} \bar{j}} & =-\nabla_{\bar{j}} T_{i k \bar{l}}-\nabla_{k} T_{\bar{j} \bar{l} \bar{i}}, \\
\nabla_{p} R_{i \bar{j} k \bar{l}}-\nabla_{i} R_{p \bar{j} k \bar{l}} & =-T_{p i}{ }^{r} R_{r \bar{j} k \bar{l}}, \\
\nabla_{\bar{q}} R_{i \bar{j} k \bar{l}}-\nabla_{\bar{j}} R_{i \bar{q} k \bar{l}} & =-T_{\bar{q} \bar{j}}{ }^{\bar{s}} R_{i \bar{s} k \bar{l}} .
\end{aligned}
$$




\section{Estimates for the parabolic flow}

Consider the following parabolic equation on a compact Hermitian manifold $(M, \hat{\omega})$ :

$$
\frac{\partial \varphi}{\partial t}=\log \frac{(\hat{\omega}+\sqrt{-1} \partial \bar{\partial} \varphi)^{n}}{\hat{\omega}^{n}}+F(\varphi, z),
$$

where $F: \mathbb{R} \times M \rightarrow \mathbb{R}$ is a smooth function and $\left.\varphi\right|_{t=0}=\varphi_{0}$ is smooth. By the theory of parabolic equations, there exists a unique smooth solution $\varphi(t)$ with $\hat{\omega}+\sqrt{-1} \partial \bar{\partial} \varphi>0$ for a short time. Denote $\dot{\varphi}$ for $\frac{\partial \varphi}{\partial t}$. We have the following proposition which generalizes the estimates in [18] to compact Hermitian manifolds.

Proposition 3.1. Given a compact Hermitian manifold $(M, \hat{\omega})$, there exists $T>0$ depending only on $\sup \left|\varphi_{0}\right|$ and $F$, such that equation (3.1) has a smooth solution $\varphi(t, z)$ on $[0, T]$. Moreover, there exist smooth functions $C_{k}(t)$ for all $k$ on $(0, T]$ depending only on $\sup \left|\varphi_{0}\right|, \sup \left|\dot{\varphi}_{0}\right|, \hat{\omega}$ and $F$ which blow up as $t \rightarrow 0$, such that

$$
\|\varphi(t)\|_{C^{k}(M)}<C_{k}(t)
$$

for $t \leq T$.

We write $g$ for the metric associated to $\omega=\hat{\omega}+\sqrt{-1} \partial \bar{\partial} \varphi$, where $\hat{\omega}$ is the background Hermitian metric on a compact complex manifold $M$. Denote $|\cdot|$ the norm of tensors with respect to $g, \nabla$ the Chern connection of $g$ and $\Delta=g^{p \bar{q}} \nabla_{p} \nabla_{\bar{q}}$ the Laplacian of $\nabla$. We use $\hat{\nabla}, \hat{R}_{i \bar{j}},|\cdot|_{\hat{g}}, \Delta_{\hat{g}}$, etc. to denote the quantities associated to $\hat{\omega}$. Throughout the section, $C, C^{\prime}, c, c_{i}, \ldots$ will be some constants which depend only on $\sup \left|\varphi_{0}\right|$, sup $\left|\dot{\varphi}_{0}\right|($ and $\hat{\omega}, F)$, and may vary from line to line. Also, we may denote $H$ to be different quantities.

First we have the following lemma from [18].

Lemma 3.1. There exist $T, C>0$ depending only on $\sup \left|\varphi_{0}\right|$ and $F$, such that

$$
|\varphi(t)|<C, \quad|\dot{\varphi}(t)| \leq \sup |\dot{\varphi}(0)| \mathrm{e}^{C t}
$$


when the solution exists and $t \leq T$. In particular,

$$
\left|\log \frac{(\hat{\omega}+\sqrt{-1} \partial \bar{\partial} \varphi)^{n}}{\hat{\omega}^{n}}\right|<C^{\prime}
$$

for some $C^{\prime}$ depending only on $\sup \left|\varphi_{0}\right|, \sup \left|\dot{\varphi}_{0}\right|$ and $F$.

The proof follows from [18, Lemma 3], as it does not need the Kähler condition. Now we can fix a $T^{\prime} \leq T$, such that there exists a smooth solution to $(3.1)$ on $\left[0, T^{\prime}\right]$.

The $C^{1}$ estimate in [18] was obtained by modifying Błocki's estimate [2] (see also [7, 15]). In Hermitian case, we need the following special local coordinate system from Guan-Li [6], which is also crucial for our secondorder estimate.

Lemma 3.2. Around a point $p \in M$, there exist local coordinates such that at $p$,

$$
\hat{g}_{i \bar{j}}=\delta_{i j}, \quad \frac{\partial \hat{g}_{i \bar{i}}}{\partial z_{j}}=0
$$

for all $i, j$.

With the above lemma, we have the following gradient estimate.

Lemma 3.3. There exists $\alpha>0$ depending on $\sup \left|\varphi_{0}\right|$ and $\sup \left|\dot{\varphi}_{0}\right|$ such that

$$
|\nabla \varphi(t)|_{\hat{g}}^{2}<\mathrm{e}^{\alpha / t}
$$

for $t \leq T^{\prime}$

Proof. Define

$$
H=t \log |\nabla \varphi(t)|_{\hat{g}}^{2}-\gamma(\varphi),
$$

where $\gamma$ is a smooth function which will be determined later. If $H$ achieves maximum on $\left[0, T^{\prime}\right] \times M$ at $t=0$, then $H$ is bounded by a constant depending on $F$ and $\sup \left|\varphi_{0}\right|$ by Lemma 3.1. Now assume that $H$ achieves its maximum at a point $\left(t_{0}, z_{0}\right), t_{0}>0$. We use $\varphi_{i}, \varphi_{i \bar{j}}, \varphi_{i \bar{j} k}$, etc. to denote partial derivatives. Choose a coordinate system around $z_{0}$ in Lemma 3.2, 
such that $\varphi_{i \bar{j}}$ is diagonal at $z_{0}$. Write $\rho=|\nabla \varphi(t)|_{\hat{g}}^{2}=\hat{g}^{i \bar{j}} \varphi_{i} \varphi_{\bar{j}}$ and $\dot{\rho}=\frac{\partial \rho}{\partial t}$. As $\left(\frac{\partial}{\partial t}-\Delta\right) H=\frac{\partial}{\partial t} H-t \frac{\Delta \rho}{\rho}+t \frac{|\nabla \rho|^{2}}{\rho^{2}}+\Delta \gamma$, we do the following calculations at $z_{0}$. First we have

$$
\begin{aligned}
\frac{\partial}{\partial t} H & =\log \rho+\frac{t \dot{\rho}}{\rho}-\gamma^{\prime} \dot{\varphi} \\
& =\log \rho-\gamma^{\prime} \dot{\varphi}+2 \frac{t}{\rho}\left(\sum_{i, k} \operatorname{Re}\left(\frac{\varphi_{k i \bar{i}} \varphi_{\bar{k}}}{1+\varphi_{i \bar{i}}}\right)+F^{\prime} \sum_{i}\left|\varphi_{i}\right|^{2}+\sum_{i} \operatorname{Re}\left(F_{i} \varphi_{\bar{i}}\right)\right)
\end{aligned}
$$

where the second equality follows from

$$
\begin{aligned}
\dot{\rho} & =\sum_{i}\left(\dot{\varphi}_{i} \varphi_{\bar{i}}+\varphi_{i} \dot{\varphi}_{\bar{i}}\right) \\
\dot{\varphi}_{i} & =g^{k \bar{l}}\left(\partial_{i} \hat{g}_{k \bar{l}}+\varphi_{i \bar{l} k}\right)-\hat{g}^{k \bar{l}} \partial_{i} \hat{g}_{k \bar{l}}+F^{\prime} \varphi_{i}+F_{i} \\
& =\sum_{k} \frac{\varphi_{i k \bar{k}}}{1+\varphi_{k \bar{k}}}+F^{\prime} \varphi_{i}+F_{i} .
\end{aligned}
$$

Here $F^{\prime}$ is the derivative in the $\varphi$ direction. Also, using $\partial_{i} \partial_{i} \hat{g}^{k \bar{l}}=-\partial_{i} \partial_{\bar{i}} \hat{g}_{l \bar{k}}+$ $\sum_{q} \partial_{i} \hat{g}_{q \bar{k}} \partial_{\bar{i}} \hat{g}_{l \bar{q}}+\sum_{p} \partial_{i} \hat{g}_{l \bar{p}} \partial_{\bar{i}} \hat{g}_{p \bar{k}}$, we get

$$
\begin{aligned}
\Delta \rho= & g^{i \bar{i}} \partial_{i} \partial_{\bar{i}}\left(\hat{g}^{k \bar{l}} \varphi_{k} \varphi_{\bar{l}}\right) \\
= & \sum_{i, k} \frac{1}{1+\varphi_{i \bar{i}}}\left(-\sum_{l} \partial_{i} \partial_{\bar{i}} \hat{g}_{l \bar{k}} \varphi_{k} \varphi_{l}+2 \operatorname{Re}\left(\varphi_{k \bar{i} i} \varphi_{\bar{k}}\right)\right. \\
& \left.+\left|\varphi_{k \bar{i}}-\sum_{l} \partial_{\bar{i}} \hat{g}_{\bar{l} k} \varphi_{l}\right|^{2}+\left|\varphi_{k i}-\sum_{l} \partial_{i} \hat{g}_{k \bar{l}} \varphi_{l}\right|^{2}\right)
\end{aligned}
$$

At $\left(t_{0}, z_{0}\right), \nabla H=0$ gives

$$
H_{i}=\frac{t}{\rho} \rho_{i}-\gamma^{\prime} \varphi_{i}=0
$$

Then

$$
\frac{|\nabla \rho|^{2}}{\rho^{2}}=\sum_{i} \frac{1}{1+\varphi_{i \bar{i}}}\left(\frac{\gamma^{\prime}}{t}\right)^{2}\left|\varphi_{i}\right|^{2}
$$


Also $\Delta \gamma(\varphi)=\sum_{i} \frac{1}{1+\varphi_{i \bar{i}}}\left(\gamma^{\prime \prime}\left|\varphi_{i}\right|^{2}+\gamma^{\prime} \varphi_{\bar{i} i}\right)$. Therefore we get

$$
\begin{aligned}
0 \leq & \left(\frac{\partial}{\partial t}-\Delta\right) H \\
= & \frac{\partial}{\partial t} H-t \frac{\Delta \rho}{\rho}+t \frac{|\nabla \rho|_{g}^{2}}{\rho^{2}}+\Delta \gamma \\
\leq & \log \rho-\gamma^{\prime} \dot{\varphi}+c t+\frac{c_{1} t}{\rho}-\sum_{i, k} \frac{t}{\rho} \frac{1}{1+\varphi_{i \bar{i}}} \\
& \times\left(\left|\varphi_{k \bar{i}}-\sum_{l} \partial_{\bar{i}} \hat{g}_{\bar{l} k} \varphi_{l}\right|^{2}+\left|\varphi_{k i}-\sum_{l} \partial_{i} \hat{g}_{k \bar{l}} \varphi_{l}\right|^{2}\right) \\
& +\sum_{i} \frac{\left|\varphi_{i}\right|^{2}}{1+\varphi_{i \bar{i}}}\left(\frac{\left(\gamma^{\prime}\right)^{2}}{t}+\gamma^{\prime \prime}\right)+\sum_{i} \frac{c_{2} t-\gamma^{\prime}}{1+\varphi_{i \bar{i}}}+n \gamma^{\prime}
\end{aligned}
$$

for some constants $c, c_{1}$ depending on $F$ and $c_{2}$ depending on the curvature of $\hat{\omega}$. Now we use the same trick in $[2,18]$ to control the term containing $\gamma^{\prime 2}$. From (3.6) we get

$$
\gamma^{\prime} \rho \varphi_{i}=t \rho_{i}=t\left(\varphi_{i} \varphi_{i \bar{i}}+\sum_{k} \varphi_{k i} \varphi_{\bar{k}}-\sum_{k, l} \partial_{i} \hat{g}_{l \bar{k}} \varphi_{k} \varphi_{\bar{l}}\right)
$$

which gives

$$
\sum_{k}\left(\varphi_{k i}-\sum_{l} \partial_{i} \hat{g}_{k \bar{l}} \varphi_{l}\right) \varphi_{\bar{k}}=t^{-1} \gamma^{\prime} \rho \varphi_{i}-\varphi_{i} \varphi_{i \bar{i}}
$$

So,

$$
\begin{aligned}
\frac{t}{\rho} \sum_{i, k} \frac{\left|\varphi_{k i}-\sum_{l} \partial_{i} \hat{g}_{k \bar{l}} \varphi_{l}\right|^{2}}{1+\varphi_{i \bar{i}}} & \geq \frac{t}{\rho^{2}} \sum_{i} \frac{\left|\sum_{k}\left(\varphi_{k i}-\sum_{l} \partial_{i} \hat{g}_{k \bar{l}} \varphi_{l}\right) \varphi_{\bar{k}}\right|^{2}}{1+\varphi_{i \bar{i}}} \\
& =\frac{t}{\rho^{2}} \sum_{i} \frac{\left|t^{-1} \gamma^{\prime} \rho \varphi_{i}-\varphi_{i} \varphi_{i \bar{i}}\right|^{2}}{1+\varphi_{i \bar{i}}} \\
& \geq \frac{\left(\gamma^{\prime}\right)^{2}}{t} \sum_{i} \frac{\left|\varphi_{i}\right|^{2}}{1+\varphi_{i \bar{i}}}-2 \gamma^{\prime},
\end{aligned}
$$


where we assume $\gamma^{\prime}>0$. As $\dot{\varphi}$ is bounded from Lemma 3.1 for $t \leq T^{\prime}$, the above estimates give

$$
0 \leq \log \rho+c t+\sum_{i} \frac{\gamma^{\prime \prime}\left|\varphi_{i}\right|^{2}}{1+\varphi_{i \bar{i}}}+\sum_{i} \frac{c_{1} t-\gamma^{\prime}}{1+\varphi_{i \bar{i}}}+(n+2+c) \gamma^{\prime}+\frac{c_{2} t}{\rho} .
$$

Take $\gamma(x)=A x-\frac{1}{A} x^{2}$. Assume that $\log \rho \geq 1$ at $\left(t_{0}, z_{0}\right)$ and choose $A$ to be sufficiently large, then we get

$$
\sum_{i} \frac{\left|\varphi_{i}\right|^{2}}{1+\varphi_{i \bar{i}}}+\sum_{i} \frac{1}{1+\varphi_{i \bar{i}}} \leq c^{\prime} \log \rho
$$

for some constant $c^{\prime}$. The above inequality together with (3.3) imply that

$$
1+\varphi_{i \bar{i}} \leq c\left(c^{\prime} \log \rho\right)^{n-1} .
$$

Then we have

$$
\rho=\sum_{i}\left|\varphi_{i}\right|^{2} \leq n c\left(c^{\prime} \log \rho\right)^{n},
$$

which shows that $\rho$ is bounded at $\left(t_{0}, z_{0}\right)$. Therefore $H$ has a bound depending only on $\sup \left|\varphi_{0}\right|, \sup \left|\dot{\varphi}_{0}\right|$ and the estimate (3.5) follows.

Now we will give the second-order estimate. We use the idea of $[6,20]$ and follow the argument in [5] closely. For local computations in the proof of the following proposition, we always use a coordinate system in Lemma 3.2 around a point $p$, such that $\hat{g}_{i \bar{j}}=\delta_{i j}, \frac{\partial \hat{g}_{i \bar{i}}}{\partial z_{j}}=0$ and $\varphi_{i \bar{j}}$ is diagonal at $p$.

Proposition 3.2. There exists $C>0$ depending on $\sup \left|\varphi_{0}\right|$ and $\sup \left|\dot{\varphi}_{0}\right|$, such that

$$
\operatorname{tr}_{\hat{g}} g=n+\Delta_{\hat{g}} \varphi(t)<\mathrm{e}^{C e^{\alpha / t}}
$$

for $t \leq T^{\prime}$, where $\alpha$ is the same as in Lemma 3.3.

Proof. Let

$$
H=\mathrm{e}^{-\frac{\alpha}{t}} \log \operatorname{tr}_{\hat{g}} g+\mathrm{e}^{\Psi},
$$

where $\Psi=A(\sup \varphi-\varphi)$ and $A$ is a constant to be chosen later. First we have

$$
\begin{aligned}
\left(\frac{\partial}{\partial t}-\Delta\right) H= & \frac{\alpha}{t^{2}} \mathrm{e}^{-\frac{\alpha}{t}} \log \operatorname{tr}_{\hat{g}} g+\frac{\mathrm{e}^{-\frac{\alpha}{t}}}{\operatorname{tr}_{\hat{g}} g} \Delta_{\hat{g}} \dot{\varphi}-A \mathrm{e}^{\Psi} \dot{\varphi} \\
& -\mathrm{e}^{-\frac{\alpha}{t}} \Delta \log \operatorname{tr}_{\hat{g}} g-A^{2}|\nabla \varphi|^{2} \mathrm{e}^{\Psi}-A\left(\operatorname{tr}_{g} \hat{g}-n\right) \mathrm{e}^{\Psi} .
\end{aligned}
$$


It follows from (3.1) that

$$
\Delta_{\hat{g}} \dot{\varphi}=-\operatorname{tr}_{\hat{g}} \operatorname{Ric}(g)+\operatorname{tr}_{\hat{g}} \operatorname{Ric}(\hat{g})+\Delta_{\hat{g}} F(\varphi, z),
$$

where

$$
\Delta_{\hat{g}} F(\varphi, z)=F^{\prime \prime}|\nabla \varphi|_{\hat{g}}^{2}+F^{\prime} \Delta_{\hat{g}} \varphi+2 \operatorname{Re}\left(g^{i \bar{j}} F_{i}^{\prime} \varphi_{j}\right)+\Delta_{\hat{g}} F
$$

Here $F^{\prime}$ is the derivative in the $\varphi$ direction, $\Delta_{\hat{g}}$ is the complex Laplacian of $F$ in the $z$ variable. Use that

$$
\begin{aligned}
\operatorname{tr}_{\hat{g}} \operatorname{Ric}(g) & =\sum_{i, k} g^{i \bar{i}}\left(-\partial_{k} \partial_{\bar{k}} g_{i \bar{i}}+g^{j \bar{j}} \partial_{k} g_{i \bar{j}} \partial_{\bar{k}} g_{j \bar{i}}\right) \\
& =\sum_{i, k} g^{i \bar{i}}\left(-\varphi_{i \bar{i} k \bar{k}}-\partial_{k} \partial_{\bar{k}} \hat{g}_{i \bar{i}}+g^{j \bar{j}} \partial_{k} g_{i \bar{j}} \partial_{\bar{k}} g_{j \bar{i}}\right)
\end{aligned}
$$

to rewrite $(3.9)$ as

$$
\begin{aligned}
\sum_{i, k} g^{i \bar{i}} \varphi_{i \bar{i} k \bar{k}}= & -\sum_{i, k} g^{i \bar{i}} \partial_{k} \partial_{\bar{k}} \hat{g}_{i \bar{i}}+\sum_{i, j, k} g^{i \bar{i}} g^{j \bar{j}} \partial_{k} g_{i \bar{j}} \partial_{\bar{k}} g_{j \bar{i}} \\
& +\Delta_{\hat{g} \dot{\varphi}} \operatorname{tr}_{\hat{g}} \operatorname{Ric}(\hat{g})-\Delta_{\hat{g}} F(\varphi, z) \\
\geq & \sum_{i, j, k} g^{i \bar{i}} g^{j \bar{j}} \partial_{k} g_{i \bar{j}} \partial_{\bar{k}} g_{j \bar{i}}+\Delta_{\hat{g}} \dot{\varphi}-C_{1}|\nabla \varphi|_{\hat{g}}^{2}-C_{2} \operatorname{tr}_{\hat{g}} g \operatorname{tr}_{g} \hat{g}
\end{aligned}
$$

From the bound in (3.3), we have $\operatorname{tr}_{\hat{g}} g, \operatorname{tr}_{g} \hat{g} \geq C^{-1}$ for some constant $C$ and then $\operatorname{tr}_{\hat{g}} g, \operatorname{tr}_{g} \hat{g} \leq C \operatorname{tr}_{g} \hat{g} \operatorname{tr}_{\hat{g}} g$. These are used in the above inequality. We will also use them frequently in the following. As the estimates in [20, (2.6)] we have

$$
\Delta \operatorname{tr}_{\hat{g}} g \geq \sum_{i, k} g^{i \bar{i}} \varphi_{i \bar{i} k \bar{k}}-2 \operatorname{Re}\left(\sum_{i, j, k} g^{i \bar{i}} \partial_{i} \hat{g}_{j \bar{k}} \varphi_{k \bar{j} i}\right)-C \operatorname{tr}_{\hat{g}} g \operatorname{tr}_{g} \hat{g}
$$

To control $\sum_{i, j, k} g^{i \bar{i}} \partial_{i} \hat{g}_{j \bar{k}} \varphi_{k \bar{j} i}$, we use a trick from [6].

$$
\sum_{i, j, k} g^{i \bar{i}} \partial_{i} \hat{g}_{j \bar{k}} \varphi_{k \bar{j} i}=\sum_{i} \sum_{j \neq k}\left(g^{i \bar{i}} \partial_{\bar{i}} \hat{g}_{j \bar{k}} \partial_{k} g_{i \bar{j}}-g^{i \bar{i}} \partial_{\bar{i}} \hat{g}_{j \bar{k}} \partial_{k} \hat{g}_{i \bar{j}}\right)
$$


So,

$$
\begin{aligned}
\mid 2 \operatorname{Re}\left(\sum_{i, j, k} g^{i \bar{i}} \partial_{i} \hat{g}_{j \bar{k}} \varphi_{k \overline{j i}} \mid\right. & \leq \sum_{i} \sum_{j \neq k}\left(g^{i \bar{i}} g^{j \bar{j}} \partial_{k} g_{i \bar{j}} \partial_{\bar{k}} g_{j \bar{i}}+g^{i \bar{i}} g_{j \bar{j}} \partial_{\bar{i}} \hat{g}_{j \bar{k}} \partial_{i} \hat{g}_{k \bar{j}}\right)+C \operatorname{tr}_{g} \hat{g} \\
& \leq \sum_{i} \sum_{j \neq k} g^{i \bar{i}} g^{j \bar{j}} \partial_{k} g_{i \bar{j}} \partial_{\bar{k}} g_{j \bar{i}}+C \operatorname{tr}_{g} \hat{g} \operatorname{tr}_{\hat{g}} g .
\end{aligned}
$$

Combining (3.10) to (3.12), we can get

$$
\Delta \operatorname{tr}_{\hat{g}} g \geq \sum_{i, j} g^{i \bar{i}} g^{j \bar{j}} \partial_{j} g_{i \bar{j}} \partial_{\bar{j}} g_{j \bar{i}}+\Delta_{\hat{g}} \dot{\varphi}-C_{1}|\nabla \varphi|_{\hat{g}}^{2}-C \operatorname{tr}_{g} \hat{g} \operatorname{tr}_{\hat{g}} g
$$

Now we will control $\frac{\left|\partial \operatorname{tr}_{\hat{g}} g\right|^{2}}{\left(\operatorname{tr}_{\hat{g}} g\right)^{2}}$. As

$$
\partial_{i} \operatorname{tr}_{\hat{g}} g=\partial_{i} \sum_{j} \varphi_{j \bar{j}}=\sum_{j} \partial_{j} \varphi_{i \bar{j}}=\sum_{j}\left(\partial_{j} g_{i \bar{j}}-\partial_{j} \hat{g}_{i \bar{j}}\right)
$$

then

$$
\begin{aligned}
\frac{\left|\partial \operatorname{tr}_{\hat{g}} g\right|^{2}}{\left(\operatorname{tr}_{\hat{g}} g\right)^{2}} & \leq \frac{1}{\left(\operatorname{tr}_{\hat{g}} g\right)^{2}} \sum_{i, j, k} g^{i \bar{i}} \partial_{j} g_{i \bar{j}} \partial_{\bar{k}} g_{k \bar{i}}-\frac{2}{\left(\operatorname{tr}_{\hat{g}} g\right)^{2}} \\
& \times \operatorname{Re}\left(\sum_{i, j, k} g^{i \bar{i}} \partial_{j} \hat{g}_{i \bar{j}} \partial_{\bar{k}} g_{k \bar{i}}\right)+C \operatorname{tr}_{g} \hat{g}
\end{aligned}
$$

Assume that $H$ achieves maximum at $\left(t_{0}, z_{0}\right), t_{0}>0$, then $\nabla H\left(t_{0}, z_{0}\right)=0$ gives

$$
\frac{\mathrm{e}^{-\frac{\alpha}{t}} \partial_{\bar{i}} \operatorname{tr}_{\hat{g}} g}{\operatorname{tr}_{\hat{g}} g}-A \mathrm{e}^{\Psi} \varphi_{\bar{i}}=0
$$

That is,

$$
\sum_{k} \partial_{\bar{i}} g_{k \bar{k}}=A \mathrm{e}^{\frac{\alpha}{t}} \operatorname{tr}_{\hat{g}} g \varphi_{i}^{-} \mathrm{e}^{\Psi}
$$

Together with $\partial_{\bar{k}} g_{k \bar{i}}=\partial_{\bar{k}} \hat{g}_{k \bar{i}}+\partial_{\bar{i}} g_{k \bar{k}}$, we get

$$
\begin{aligned}
\left|\frac{2}{\left(\operatorname{tr}_{\hat{g}} g\right)^{2}} \operatorname{Re}\left(\sum_{i, j, k} g^{i \bar{i}} \partial_{j} \hat{g}_{i \bar{j}} \partial_{\bar{k}} g_{k \bar{i}}\right)\right| & \leq\left|\frac{2 A \mathrm{e}^{\frac{\alpha}{t}} \mathrm{e}^{\Psi}}{\operatorname{tr}_{\hat{g}} g} \operatorname{Re} \sum_{i, j} g^{i \bar{i}} \partial_{j} \hat{g}_{i \bar{j}} \varphi_{\bar{i}}\right|+C \operatorname{tr}_{g} \hat{g} \\
& \leq \mathrm{e}^{\frac{\alpha}{t}} \mathrm{e}^{\Psi}\left(A^{2}|\nabla \varphi|^{2}+\frac{C \operatorname{tr}_{g} \hat{g}}{\left(\operatorname{tr}_{\hat{g}} g\right)^{2}}\right)+C \operatorname{tr}_{g} \hat{g} \\
& \leq \mathrm{e}^{\frac{\alpha}{t}} \mathrm{e}^{\Psi}\left(A^{2}|\nabla \varphi|^{2}+C^{\prime} \operatorname{tr}_{g} \hat{g}\right)+C \operatorname{tr}_{g} \hat{g} .
\end{aligned}
$$


Using the Cauchy-Schwarz inequality as in Yau's second-order estimate [25] (see Equation (2.21) in [20]), we have

$$
\frac{1}{\operatorname{tr}_{\hat{g}} g} \sum_{i, j, k} g^{i \bar{i}} \partial_{j} g_{i \bar{j}} \partial_{\bar{k}} g_{k \bar{i}} \leq \sum_{i, j} g^{i \bar{i}} g^{j \bar{j}} \partial_{j} g_{i \bar{j}} \partial_{\bar{j}} g_{j \bar{i}}
$$

Combining (3.13) and (3.14), we get

$$
\frac{\left|\partial \operatorname{tr}_{\hat{g}} g\right|^{2}}{\left(\operatorname{tr}_{\hat{g}} g\right)^{2}} \leq \frac{1}{\operatorname{tr}_{\hat{g}} g} \sum_{i, j} g^{i \bar{i}} g^{j \bar{j}} \partial_{j} g_{i \bar{j}} \partial_{\bar{j}} g_{j \bar{i}}+\mathrm{e}^{\frac{\alpha}{t}} \mathrm{e}^{\Psi}\left(A^{2}|\nabla \varphi|^{2}+C^{\prime} \operatorname{tr}_{g} \hat{g}\right)+C \operatorname{tr}_{g} \hat{g}
$$

So,

$$
\begin{aligned}
\mathrm{e}^{-\frac{\alpha}{t} \Delta \log \operatorname{tr}_{\hat{g}} g=} & \mathrm{e}^{-\frac{\alpha}{t}}\left(\frac{\Delta \operatorname{tr}_{\hat{g}} g}{\operatorname{tr}_{\hat{g}} g}-\frac{\left|\partial \operatorname{tr}_{\hat{g}} g\right|^{2}}{\left(\operatorname{tr}_{\hat{g}} g\right)^{2}}\right) \\
\geq & \frac{\mathrm{e}^{-\frac{\alpha}{t}}}{\operatorname{tr}_{\hat{g}} g} \Delta_{\hat{g}} \dot{\varphi}-C_{1} \frac{\mathrm{e}^{-\frac{\alpha}{t}}}{\operatorname{tr}_{\hat{g}} g}|\nabla \varphi|_{\hat{g}}^{2}-C \mathrm{e}^{-\frac{\alpha}{t}} \operatorname{tr}_{g} \hat{g} \\
& -A^{2}|\nabla \varphi|^{2} \mathrm{e}^{\Psi}-C^{\prime} \operatorname{tr}_{g} \hat{g} \mathrm{e}^{\Psi}
\end{aligned}
$$

From (3.3) we have $\operatorname{tr}_{\hat{g}} g \leq C\left(\operatorname{tr}_{g} \hat{g}\right)^{n-1}$ for some constant $C$. Now putting (3.15) into (3.8) and using (3.5) and that $\varphi, \dot{\varphi}$ are bounded, we have

$$
\begin{aligned}
\left(\frac{\partial}{\partial t}-\Delta\right) H \leq & C \log \operatorname{tr}_{\hat{g}} g-A \dot{\varphi} \mathrm{e}^{\Psi}+C_{1}+C \mathrm{e}^{-\frac{\alpha}{t}} \operatorname{tr}_{g} \hat{g} \\
& +C \operatorname{tr}_{g} \hat{g} \mathrm{e}^{\Psi}+A n \mathrm{e}^{\Psi}-A \operatorname{tr}_{g} \hat{g} \mathrm{e}^{\Psi} \\
\leq & C^{\prime} \log \operatorname{tr}_{\hat{g}} g+A C^{\prime} \mathrm{e}^{\Psi}-(A-C) \mathrm{e}^{\Psi} \operatorname{tr}_{g} \hat{g}+C \mathrm{e}^{-\frac{\alpha}{t}} \operatorname{tr}_{g} \hat{g} \\
\leq & -\left(A-C-C_{1}\right) e^{\Psi} \operatorname{tr}_{g} \hat{g}+A C^{\prime} \mathrm{e}^{\Psi} .
\end{aligned}
$$

Choosing $A$ large enough such that $A-C-C_{1} \geq 0$, then at $\left(t_{0}, z_{0}\right)$,

$$
0 \leq-\left(A-C-C_{1}\right) \operatorname{tr}_{g} \hat{g}+A C^{\prime}
$$

for $t \leq T^{\prime}$ gives $\operatorname{tr}_{g} \hat{g} \leq C^{\prime}$ at $\left(t_{0}, z_{0}\right)$, which implies that $H \leq C$ for some constant $C$ depending on $\sup \left|\varphi_{0}\right|$ and $\sup \left|\dot{\varphi}_{0}\right|$. Then we obtain the desired estimate (3.7).

Now we give the third-order estimate. Our proof is based on the arguments in [14, 19] (see also [24]). As in [25], consider $S=g^{i \bar{p}} g^{q \bar{j}} g^{k \bar{r}} \hat{\nabla}_{k} \varphi_{i \bar{j}} \hat{\nabla}_{\bar{r}} \varphi_{\bar{p} q}$. 
We introduce the tensor $\Phi_{i j}{ }^{k}=\Gamma_{i j}^{k}-\hat{\Gamma}_{i j}^{k}$ and then

$$
S=|\Phi|^{2}=g^{i \bar{p}} g^{j \bar{q}} g_{k \bar{r}} \Phi_{i j}{ }^{k} \Phi_{\bar{p} \bar{q}} \overline{\bar{q}} .
$$

From now on, we will write $k(t), k_{1}(t), k_{2}(t), \ldots$ for functions of the form $K \mathrm{e}^{\lambda C \mathrm{e}^{\alpha / t}}$ where $\mathrm{e}^{C \mathrm{e}^{\alpha / t}}$ is the bound in Proposition 3.2, and $K, \lambda$ are constants depending only on $\hat{\omega}$ and $F$. In the proof of the following proposition, we will use the estimates $|\nabla \varphi(t)|_{\hat{g}}^{2} \leq k(t), \operatorname{tr}_{\hat{g}} g \leq k(t)$ repeatedly.

Proposition 3.3. There exists a smooth function $C(t)>0$ on $\left(0, T^{\prime}\right]$ depending only on $\sup \left|\varphi_{0}\right|, \sup \left|\dot{\varphi}_{0}\right|$ and blowing up as $t \rightarrow 0$, such that $S<C(t)$ for $t \leq T^{\prime}$.

Proof. We write $\Delta=g^{p \bar{q}} \nabla_{p} \nabla_{\bar{q}}$. As the calculations in $[14,19]$, first we have

$$
\begin{aligned}
\Delta S= & |\bar{\nabla} \Phi|^{2}+|\nabla \Phi|^{2}-\Phi_{i j}{ }^{k}\left(R_{p k}^{p q} \Phi_{q}^{i j}-R_{p}^{p}{ }_{q}^{i} \Phi_{k}^{q j}-R_{p}^{p}{ }_{q}^{j} \Phi_{k}^{i q}\right) \\
& +2 \operatorname{Re}\left(\Delta \Phi_{i j}{ }^{k} \Phi_{k}^{i j}\right), \\
\frac{\partial}{\partial t} S= & \Phi_{i j}{ }^{k}\left(\frac{\partial}{\partial t} g^{i \bar{q}} \Phi_{\bar{q} k}^{j}+\frac{\partial}{\partial t} g_{k \bar{q}} \Phi^{i j \bar{q}}+\frac{\partial}{\partial t} g^{j \bar{q}} \Phi^{i}{ }_{\bar{q} k}\right)+2 \operatorname{Re}\left(\frac{\partial}{\partial t} \Phi_{i j}{ }^{k} \Phi_{k}^{i j}\right) \\
= & \Phi_{i j}{ }^{k}\left(g^{q \bar{r}} \frac{\partial}{\partial t} g_{k \bar{r}} \Phi^{i j}{ }_{q}-g^{i \bar{r}} \frac{\partial}{\partial t} g_{q \bar{r}} \Phi_{k}^{q j}-g^{j \bar{r}} \frac{\partial}{\partial t} g_{q \bar{r}} \Phi_{k}^{i q}\right) \\
& +2 \operatorname{Re}\left(\frac{\partial}{\partial t} \Phi_{i j}{ }^{k} \Phi_{k}^{i j}\right) .
\end{aligned}
$$

Thus,

$$
\begin{aligned}
\left(\frac{\partial}{\partial t}-\Delta\right) S= & -|\bar{\nabla} \Phi|^{2}-|\nabla \Phi|^{2}+\Phi_{i j}{ }^{k}\left(B_{k}{ }^{q} \Phi^{i j}{ }_{q}-B_{q}{ }^{i} \Phi_{k}^{q j}{ }_{k}-B_{q}{ }^{j} \Phi_{k}^{i q}{ }_{k}\right) \\
& +2 \operatorname{Re}\left(\left(\frac{\partial}{\partial t}-\Delta\right) \Phi_{i j}{ }^{k} \Phi_{k}^{i j}\right)
\end{aligned}
$$

where $B_{i}^{j}=g^{j \bar{r}} \frac{\partial}{\partial t} g_{i \bar{r}}+R_{p i}^{p}{ }_{i}^{j}$. From Equation (3.1) and Formula (2.2), we get

$$
\begin{aligned}
& \frac{\partial}{\partial t} g_{i \bar{j}}=-R_{i \bar{j}}+\hat{R}_{i \bar{j}}+F_{i \bar{j}}(\varphi, z), \\
& R_{p k}^{p q}=R_{k}^{q}-\nabla^{p} T_{p k}^{q}-\nabla_{k} T_{p}^{p q} .
\end{aligned}
$$

Hence

$$
B_{i}^{j}=g^{j \bar{r}}\left(\hat{R}_{i \bar{r}}+F_{i \bar{r}}(\varphi, z)\right)-\nabla^{p} T_{p i}{ }^{j}-\nabla_{i} T_{p}^{p j}
$$


Here $F_{i \bar{r}}(\varphi, z)=F_{i \bar{r}}+F^{\prime \prime} \varphi_{i} \varphi_{\bar{r}}+F^{\prime} \varphi_{i \bar{r}}+F_{i}^{\prime} \varphi_{\bar{r}}+F_{\bar{r}}^{\prime} \varphi_{i}$.

Now we compute the evolution of $\Phi_{i j}{ }^{k}$. First,

$$
\begin{aligned}
\frac{\partial}{\partial t} \Phi_{i j}{ }^{k} & =g^{k \bar{l}} \nabla_{i} \frac{\partial}{\partial t} g_{j \bar{l}} \\
& =-\nabla_{i} R_{j}{ }^{k}+g^{k \bar{l}}\left(\nabla_{i} \hat{R}_{j \bar{l}}+\nabla_{i} F_{j \bar{l}}(\varphi, z)\right) .
\end{aligned}
$$

Note that

$$
\nabla_{\bar{q}} \Phi_{i j}{ }^{k}=-R_{i \bar{q} j}{ }^{k}+\hat{R}_{i \bar{q} j}{ }^{k}
$$

Then

$$
\begin{aligned}
\Delta \Phi_{i j}{ }^{k} & =-\nabla^{\bar{p}} R_{i \bar{p} j}{ }^{k}+\nabla^{\bar{p}} \hat{R}_{i \bar{p} j}{ }^{k} \\
& =\nabla_{i}\left(-R_{j}{ }^{k}+\nabla^{q} T_{q j}{ }^{k}+\nabla_{j} T_{p}^{p k}\right)-T_{i q}{ }^{r} R_{r}{ }^{q k}{ }^{k}+\nabla^{\bar{p}} \hat{R}_{i \bar{p} j}{ }^{k} .
\end{aligned}
$$

So we have

$$
\begin{aligned}
\left(\frac{\partial}{\partial t}-\Delta\right) \Phi_{i j}{ }^{k}= & \nabla_{i}\left(g^{k \bar{l}}\left(\hat{R}_{j \bar{l}}+F_{j l}(\varphi, z)\right)-\nabla^{q} T_{q j}{ }^{k}-\nabla_{j} T_{p}^{p k}\right) \\
& +T_{i q}^{r} R_{r}^{q k}{ }_{j}^{k}-\nabla^{\bar{p}} \hat{R}_{i \bar{p} j}{ }^{k} \\
= & \nabla_{i} B_{j}{ }^{k}+T_{i q}^{r} R_{r j}^{q k}-\nabla^{\bar{p}} \hat{R}_{i \bar{p} j}{ }^{k} .
\end{aligned}
$$

Combining with (3.16) we get

$$
\begin{aligned}
\left(\frac{\partial}{\partial t}-\Delta\right) S= & -|\bar{\nabla} \Phi|^{2}-|\nabla \Phi|^{2}+\Phi_{i j}{ }^{k}\left(B_{k}{ }^{q} \Phi^{i j}{ }_{q}-B_{q}{ }^{i} \Phi_{k}^{q j}{ }_{k}-B_{q}{ }^{j} \Phi_{k}^{i q}\right) \\
& +2 \operatorname{Re}\left(\nabla_{i} B_{j}{ }^{k}+T_{i q}{ }^{r} R_{r}{ }^{q k}{ }^{k}-\nabla^{\bar{p}} \hat{R}_{i \bar{p} j}{ }^{k}\right) \Phi_{k}^{i j}{ }_{k} .
\end{aligned}
$$

As $T_{i j \bar{k}}=\hat{T}_{i j \bar{k}}$,

$$
\nabla^{p} T_{p k}^{q}=g^{p \bar{l}} g^{q \bar{r}}\left(\hat{\nabla}_{\bar{l}} \hat{T}_{p k \bar{r}}-\Phi_{\bar{l} \bar{r}}{ }^{\bar{s}} \hat{T}_{p k \bar{s}}\right)
$$

By $(3.17)$

$$
\left|B_{i \bar{j}}\right| \leq k(t)\left(S^{1 / 2}+1+|\nabla \varphi|_{g}^{2}+\left|\varphi_{i \bar{j}}\right|_{g}^{2}\right) \leq k(t)\left(S^{1 / 2}+1\right)
$$


Now we want to control $\nabla_{i} B_{j}{ }^{k}$. From (3.17) we need the following estimates from [19] obtained by similar calculations as (3.19):

$$
\begin{aligned}
& \left|\nabla_{i} \nabla^{q} T_{q j}{ }^{k}\right| \leq k(t)(S+|\bar{\nabla} \Phi|+1), \\
& \left|\nabla_{i} \nabla_{j} T_{\bar{p}}{ }^{k \bar{p}}\right| \leq k(t)(S+|\nabla \Phi|+1) .
\end{aligned}
$$

Also

$$
\begin{aligned}
& \left|T_{i q}^{r} R_{r j}^{q k}\right| \leq k(t)(|\bar{\nabla} \Phi|+1), \\
& \left|\nabla^{\bar{p}} R_{\bar{p} i j}{ }^{k}\right| \leq k(t)\left(S^{1 / 2}+1\right) .
\end{aligned}
$$

We bound the terms with $\varphi_{i j}$ and $\Phi_{k}^{i j}$ in $\operatorname{Re}\left(\nabla_{i} B_{j}{ }^{k} \Phi_{k}^{i j}\right)$ by $\left|\varphi_{i j}\right|^{2}+k(t) S$. Together with the above estimates we get

$$
\left(\frac{\partial}{\partial t}-\Delta\right) S \leq k(t)\left(S^{3 / 2}+S+1\right)+\sum_{i, j}\left|\varphi_{i j}\right|^{2}-\frac{1}{2}\left(|\nabla \Phi|^{2}+|\bar{\nabla} \Phi|^{2}\right)
$$

We will use a similar way as in $[16,19]$ to control the term $S^{3 / 2}$. The evolution equations below can be obtained by following the computations in $[18,19]$ :

$$
\begin{gathered}
\left(\frac{\partial}{\partial t}-\Delta\right) \operatorname{tr}_{\hat{g}} g \leq-\frac{S}{k_{2}(t)}+k_{2}(t) \\
\left(\frac{\partial}{\partial t}-\Delta\right)|\nabla \varphi|_{\hat{g}}^{2} \leq-\sum_{i, j} \frac{\left|\varphi_{i j}\right|^{2}}{k_{3}(t)}+k_{3}(t)
\end{gathered}
$$

Now we will apply a maximum principle argument to the quantity

$$
H=\frac{S}{\left(C_{1}(t)-\operatorname{tr}_{\hat{g}} g\right)^{2}}+\frac{\operatorname{tr}_{\hat{g}} g}{C_{2}(t)}+\frac{|\nabla \varphi|_{\hat{g}}^{2}}{C_{3}(t)}
$$

Here we can take $C_{i}(t)$ to be the form of $L \mathrm{e}^{\lambda C \mathrm{e}^{\alpha / t}}$, where $C, \alpha$ are the same as in (3.7) and $L, \lambda$ will be determined later. Let $L, \lambda>2$ such that

$$
\frac{C_{1}(t)}{2} \leq C_{1}(t)-\operatorname{tr}_{\hat{g}} g \leq C_{1}(t), \quad 0<-\frac{C_{i}^{\prime}(t)}{C_{i}^{2}(t)} \leq \frac{1}{\sqrt{C_{i}(t)}}, \quad i=1,2,3 .
$$


We calculate the evolution of $H$.

$$
\begin{aligned}
\left(\frac{\partial}{\partial t}-\Delta\right) H= & \frac{1}{\left(C_{1}(t)-\operatorname{tr}_{\hat{g}} g\right)^{2}}\left(\frac{\partial}{\partial t}-\Delta\right) S \\
& +\frac{2 S}{\left(C_{1}(t)-\operatorname{tr}_{\hat{g}} g\right)^{3}}\left(\frac{\partial}{\partial t}-\Delta\right) \operatorname{tr}_{\hat{g}} g \\
& -\frac{4 \operatorname{Re} \nabla \operatorname{tr}_{\hat{g}} g \cdot \bar{\nabla} S}{\left(C_{1}(t)-\operatorname{tr}_{\hat{g}} g\right)^{3}}-\frac{6 S\left|\nabla \operatorname{tr}_{\hat{g}} g\right|^{2}}{\left(C_{1}(t)-\operatorname{tr}_{\hat{g}} g\right)^{4}}-\frac{2 C_{1}^{\prime}(t) S}{\left(C_{1}(t)-\operatorname{tr}_{\hat{g}} g\right)^{3}} \\
& +\frac{1}{C_{2}(t)}\left(\frac{\partial}{\partial t}-\Delta\right) \operatorname{tr}_{\hat{g}} g+\frac{1}{C_{3}(t)}\left(\frac{\partial}{\partial t}-\Delta\right)|\nabla \varphi|_{\hat{g}}^{2} \\
& -\frac{C_{2}^{\prime}(t) \operatorname{tr}_{\hat{g}} g}{C_{2}(t)^{2}}-\frac{C_{3}^{\prime}(t)|\nabla \varphi|_{\hat{g}}^{2}}{C_{3}(t)^{2}}
\end{aligned}
$$

Taking $C_{2}(t), C_{3}(t)$ large enough and using (3.5), (3.7) and (3.22), the last two terms can be bounded by a constant $C$. Assuming $S>1$ at the maximum point of $H$, from (3.20) we have

$$
\left(\frac{\partial}{\partial t}-\Delta\right) S \leq k_{1}(t)\left(S^{3 / 2}+1\right)+\sum_{i, j}\left|\varphi_{i j}\right|^{2}-\frac{1}{2}|\bar{\nabla} \Phi|^{2}
$$

Together with (3.21) and (3.22), we get

$$
\begin{aligned}
0 \leq & \left(\frac{\partial}{\partial t}-\Delta\right) H \\
\leq & \left(\frac{4 k_{1}(t)}{C_{1}^{2}(t)} S^{3 / 2}+\frac{4 k_{1}(t)}{C_{1}^{2}(t)}+\frac{4}{C_{1}^{2}(t)} \sum_{i, j}\left|\varphi_{i j}\right|^{2}-\frac{|\bar{\nabla} \Phi|^{2}}{2 C_{1}^{2}(t)}\right) \\
& +\left(-\frac{2 S^{2}}{k_{2}(t) C_{1}^{3}(t)}+\frac{16 k_{2}(t) S}{C_{1}^{3}(t)}\right) \\
& +\frac{4\left|\operatorname{Re}_{\operatorname{tr}} g \cdot \bar{\nabla} S\right|}{\left(C_{1}(t)-\operatorname{tr}_{\hat{g}} g\right)^{3}}+\frac{2 S}{\sqrt{C_{1}^{3}(t)}}+\left(-\frac{1}{k_{2}(t) C_{2}(t)} S+\frac{k_{2}(t)}{C_{2}(t)}\right) \\
& +\left(-\frac{1}{k_{3}(t) C_{3}(t)} \sum_{i, j}\left|\varphi_{i j}\right|^{2}+\frac{k_{3}(t)}{C_{3}(t)}\right)+C .
\end{aligned}
$$


As $\left|\nabla \operatorname{tr}_{\hat{g}} g\right| \leq \frac{1}{64} k_{5}(t) S^{1 / 2}$ and $|\bar{\nabla} S| \leq 2 S^{1 / 2}|\bar{\nabla} \Phi|$

$$
\frac{4\left|\operatorname{Re} \nabla \operatorname{tr}_{\hat{g}} g \cdot \bar{\nabla} S\right|}{\left(C_{1}(t)-\operatorname{tr}_{\hat{g}} g\right)^{3}} \leq \frac{k_{5}(t) S|\bar{\nabla} \Phi|}{C_{1}^{3}(t)} \leq \frac{|\bar{\nabla} \Phi|^{2}}{2 C_{1}^{2}(t)}+\frac{k_{5}^{2}(t) S^{2}}{2 C_{1}^{4}(t)}
$$

We will also use

$$
\frac{4 k_{1}(t) S^{3 / 2}}{C_{1}^{2}(t)} \leq \frac{S^{2}}{k_{2}(t) C_{1}^{3}(t)}+\frac{4 k_{1}^{2}(t) k_{2}(t) S}{C_{1}(t)}
$$

Recall that all $k_{i}(t), C_{i}(t)$ are functions of the form $L \mathrm{e}^{\lambda C \mathrm{e}^{\alpha / t}}$. First, choose $C_{i}(t)>k_{i}(t)$, then fix $C_{2}(t), C_{3}(t)$. Now take the constant $L, \lambda$ in $C_{1}(t)$ to be large enough, such that $\frac{k_{5}^{2}(t)}{2 C_{1}^{4}(t)} \leq \frac{1}{k_{2}(t) C_{1}^{3}(t)}, \frac{4}{C_{1}^{2}(t)} \leq \frac{1}{k_{3}(t) C_{3}(t)}$ and $\frac{16 k_{2}(t)}{C_{1}^{3}(t)}+$ $\frac{2}{\sqrt{C_{1}^{3}}(t)}+\frac{4 k_{1}^{2}(t) k_{2}(t)}{C_{1}(t)} \leq \frac{1}{2 k_{2}(t) C_{2}(t)}$. The above estimates then give that at $\left(t_{0}, z_{0}\right)$

$$
0 \leq \frac{-1}{2 k_{2}(t) C_{2}(t)} S+C^{\prime}
$$

for some constant $C^{\prime}$. Therefore $S \leq 4 C^{\prime} k_{2}(t) C_{2}(t) \leq C^{\prime} C_{1}(t)$ at $\left(t_{0}, z_{0}\right)$. It follows that $H$ is bounded by some constant $C$ depending only on sup $\left|\varphi_{0}\right|$ and $\sup \left|\dot{\varphi}_{0}\right|$, which gives the desired estimate of $S$.

Using (3.7), the above estimate $S \leq C(t)$ implies that $\|\varphi(t)\|_{C^{2+\alpha}(M, \hat{g})}$ can be bounded by a smooth function $C(t)$ on $\left(0, T^{\prime}\right]$, which depends only on $\sup \left|\varphi_{0}\right|$ and $\sup \left|\dot{\varphi}_{0}\right|$. Differentiating the Equation (3.1) in $t$, we get

$$
\frac{\partial \dot{\varphi}}{\partial t}=\triangle \dot{\varphi}+F^{\prime}(\varphi, z) \dot{\varphi}
$$

To apply parabolic Schauder estimates to obtain higher-order estimates, we still need to bound the derivatives of $g_{i \bar{j}}$ in the $t$-direction. Then it is sufficient to bound $|\operatorname{Ric}(g)|$.

Lemma 3.4. There exists a smooth function $C(t)>0$ on $\left(0, T^{\prime}\right]$ depending only on $\sup \left|\varphi_{0}\right|$ and $\sup \left|\dot{\varphi}_{0}\right|$ and blowing up as $t \rightarrow 0$, such that $|\operatorname{Ric}|<C(t)$ for $t \leq T^{\prime}$. 
Proof. To compute the evolution of $\mid$ Ric $\mid$, first

$$
\frac{\partial}{\partial t} R_{j \bar{k}}=-g^{l \bar{q}} \nabla_{\bar{k}} \nabla_{j} \frac{\partial}{\partial t} g_{l \bar{q}}=-g^{l \bar{q}} \nabla_{\bar{k}} \nabla_{j}\left(-R_{l \bar{q}}+\hat{R}_{l \bar{q}}+F_{l \bar{q}}(\varphi, z)\right)
$$

Using (2.1) and (2.2), we have

$$
\begin{aligned}
\nabla_{\bar{k}} \nabla_{j} R_{l \bar{q}}= & \nabla_{l} \nabla_{\bar{q}} R_{j \bar{k}}-\nabla_{l} T_{\bar{k} \bar{q}}{ }^{\bar{s}} R_{j \bar{s}}+T_{\bar{k} \bar{q}}{ }^{\bar{s}} \nabla_{l} R_{j \bar{s}}+R_{l \bar{k} j}{ }^{r} R_{r \bar{q}} \\
& -R_{l \bar{k} \bar{q}} R_{j \bar{s}}+\nabla_{\bar{k}} T_{l j}^{r} R_{r \bar{q}}+T_{l j}^{r} \nabla_{\bar{k}} R_{r \bar{q}} .
\end{aligned}
$$

So

$$
\begin{aligned}
\left(\frac{\partial}{\partial t}-\Delta\right) R_{j \bar{k}}= & \nabla_{\bar{k}} T_{l j}^{r} R_{r}^{l}+T_{l j}^{r} \nabla_{\bar{k}} R_{r}^{l}+R_{l \bar{k} j}{ }^{r} R_{r}^{l} \\
& -R_{l \bar{k}}{ }^{\bar{s} l} R_{j \bar{s}}+\nabla^{\bar{q}} T_{\bar{k} \bar{q}}{ }^{\bar{s}} R_{j \bar{s}}+T_{\bar{k} \bar{q}}{ }^{\bar{s}} \nabla^{\bar{q}} R_{j \bar{s}} \\
& -g^{l \bar{q}} \nabla_{\bar{k}} \nabla_{j}\left(\hat{R}_{l \bar{q}}+F_{l \bar{q}}(\varphi, z)\right) .
\end{aligned}
$$

From (3.19) we get

$$
|\nabla T| \leq k(t)\left(1+S^{1 / 2}\right), \quad|\bar{\nabla} T| \leq k(t)\left(1+S^{1 / 2}\right)
$$

where $S$ is bounded by some $k(t)$ by Proposition 3.3. Note that (3.18) gives

$$
R_{i \bar{j}}=\hat{R}_{i \bar{j}}+\nabla_{\bar{j}} \Phi_{i k}^{k}, \quad|\bar{\nabla} \Phi| \leq|\mathrm{Rm}|+k(t)
$$

Use this and similar calculation as (3.19) to get

$$
\left|g^{l \bar{q}} \nabla_{\bar{k}} \nabla_{j} \hat{R}_{l \bar{q}}\right| \leq k(t)(|\mathrm{Rm}|+1) .
$$

Also we have

$$
\left|g^{l \bar{q}} \nabla_{\bar{k}} \nabla_{j} F_{l \bar{q}}(\varphi, z)\right| \leq k(t)(|\mathrm{Rm}|+1) .
$$

Therefore

$$
\begin{aligned}
\left|\left(\frac{\partial}{\partial t}-\Delta\right) R_{j \bar{k}}\right| & \leq k(t)\left(|\nabla \mathrm{Ric}|+|\mathrm{Rm}|^{2}+|\mathrm{Rm}|+1\right) \\
& \leq k(t)\left(|\nabla \mathrm{Ric}|+|\mathrm{Rm}|^{2}+1\right)
\end{aligned}
$$


As $\left|\frac{\partial}{\partial t} g_{i \bar{j}}\right|=\left|-R_{i \bar{j}}+\hat{R}_{i \bar{j}}+F_{i \bar{j}}(\varphi, z)\right| \leq \mid$ Ric $\mid+k(t)$, direct computation gives

$$
\begin{aligned}
\left(\frac{\partial}{\partial t}-\Delta\right)|\operatorname{Ric}|^{2} & \leq k(t)\left(|\operatorname{Ric}|^{3}+|\operatorname{Ric}|^{2}\right) \\
& +2\left|\left(\frac{\partial}{\partial t}-\Delta\right) \operatorname{Ric}\right||\operatorname{Ric}|-2|\nabla \operatorname{Ric}|^{2} .
\end{aligned}
$$

We then obtain the following:

$$
\begin{aligned}
\left(\frac{\partial}{\partial t}-\Delta\right)|\operatorname{Ric}| & \left.=\frac{1}{2|\operatorname{Ric}|}\left(\frac{\partial}{\partial t}-\Delta\right)|\operatorname{Ric}|^{2}+\left.2|\nabla| \operatorname{Ric}\right|^{2}\right) \\
& \leq k_{1}(t)\left(|\nabla \operatorname{Ric}|+|\operatorname{Rm}|^{2}+1\right)-\frac{|\nabla \operatorname{Ric}|^{2}}{|\operatorname{Ric}|}+\frac{|\nabla| \operatorname{Ric}||^{2}}{|\operatorname{Ric}|} .
\end{aligned}
$$

Let us consider

$$
H=\frac{\mid \text { Ric } \mid}{C_{1}(t)}+\frac{S}{C_{2}(t)}
$$

as in [18] where $C_{1}(t), C_{2}(t)$ are the functions of the form $L \mathrm{e}^{\lambda C \mathrm{e}^{\alpha / t}}$ as in the proof of Propostion 3.3, such that $-\frac{C_{i}^{\prime}(t)}{C_{i}^{2}(t)} \leq \frac{1}{\sqrt{C_{i}(t)}}, i=1,2$. Assume that $H$ achieves maximum at a point $\left(t_{0}, z_{0}\right), t_{0}>0$, and assume $\mid$ Ric $\mid \geq 1$ at $\left(t_{0}, z_{0}\right)$. From (3.20) and Propositions 3.2 and 3.3 we have

$$
\left(\frac{\partial}{\partial t}-\Delta\right) S \leq-\frac{1}{2} Q+k_{2}(t)
$$

where $Q=|\nabla \Phi|^{2}+|\bar{\nabla} \Phi|^{2}$. Take $C_{1}(t)>k_{1}(t), C_{2}(t) \geq \max \left\{S, S^{2}, k_{2}(t)\right\}$. Direct computation gives

$$
\begin{aligned}
\left(\frac{\partial}{\partial t}-\Delta\right) H \leq & \frac{k_{1}(t)\left(\mid \nabla \text { Ric }\left.|+| \operatorname{Rm}\right|^{2}\right)}{C_{1}(t)}-\frac{\mid \nabla \text { Ric }\left.\right|^{2}}{C_{1}(t)|\operatorname{Ric}|}+\frac{\left.|\nabla| \operatorname{Ric}\right|^{2}}{C_{1}(t)|\operatorname{Ric}|} \\
& +\frac{|\operatorname{Ric}|}{\sqrt{C_{1}(t)}}+\left(-\frac{Q}{2 C_{2}(t)}+\frac{k_{2}(t)}{C_{2}(t)}\right)+\frac{S}{\sqrt{C_{2}(t)}} \\
\leq & \frac{k_{3}(t)|\operatorname{Rm}|^{2}}{C_{1}(t)}-\frac{\mid \nabla \text { Ric }\left.\right|^{2}}{2 C_{1}(t)|\operatorname{Ric}|}+\frac{\left.|\nabla| \operatorname{Ric}\right|^{2}}{C_{1}(t)|\operatorname{Ric}|}-\frac{Q}{2 C_{2}(t)}+C,
\end{aligned}
$$

where the last inequality we use

$$
\frac{k_{1}(t)|\nabla \operatorname{Ric}|}{C_{1}(t)} \leq \frac{\mid \nabla \text { Ric }\left.\right|^{2}}{2 C_{1}(t)|\operatorname{Ric}|}+\frac{k_{1}^{2}(t)|\operatorname{Ric}|}{2 C_{1}(t)} .
$$


Using $\nabla H=0$ at $\left(t_{0}, z_{0}\right)$ and $|\nabla| \operatorname{Ric}|| \leq \mid \nabla$ Ric $\mid$, we get

$$
\begin{aligned}
\frac{\left.|\nabla| \mathrm{Ric}\right|^{2}}{C_{1}(t)|\mathrm{Ric}|} & =\frac{|\nabla S \cdot \bar{\nabla}| \mathrm{Ric} \|}{C_{2}(t)|\mathrm{Ric}|} \\
& \leq \frac{|\nabla \mathrm{Ric}|^{2}}{2 C_{1}(t)|\operatorname{Ric}|}+\frac{C_{1}(t)|\nabla S|^{2}}{2 C_{2}^{2}(t)|\operatorname{Ric}|} \\
& \leq \frac{|\nabla \mathrm{Ric}|^{2}}{2 C_{1}(t)|\operatorname{Ric}|}+\frac{C_{1}(t) k_{4}(t) Q}{C_{2}^{2}(t)|\operatorname{Ric}|}
\end{aligned}
$$

where the last inequality we use $|\nabla S|^{2} \leq 2 S\left(|\bar{\nabla} \Phi|^{2}+|\nabla \Phi|^{2}\right)$. From (3.18),

$$
|\operatorname{Rm}|^{2} \leq \frac{3}{2} Q+k_{5}(t), \quad|\operatorname{Ric}| \leq \sqrt{Q}+k_{6}(t)
$$

Choose $C_{2}(t) \geq 8 k_{4}(t)$. Fix $C_{2}(t)$ and choose $C_{1}(t) \geq \max \left\{k_{3}(t) k_{5}(t), k_{6}(t)\right\}$ large enough such that $\frac{3 k_{3}(t)}{2 C_{1}(t)} \leq \frac{1}{4 C_{2}(t)}$ and then fix $C_{1}(t)$. Combining the above estimates, we obtain that at $\left(x_{0}, t_{0}\right)$,

$$
0 \leq\left(\frac{\partial}{\partial t}-\Delta\right) H \leq-\frac{Q}{4 C_{2}(t)}+\frac{C_{1}(t) Q}{8 C_{2}(t)|\operatorname{Ric}|}+C^{\prime}
$$

for some constant $C^{\prime}$. If $\frac{\mid \text { Ric } \mid}{C_{1}(t)} \leq 1$, then $H \leq 2$ at $\left(t_{0}, z_{0}\right)$ and we obtain the estimate for $\mid$ Ric $\mid$. Otherwise at $\left(t_{0}, z_{0}\right)$

$$
0 \leq-\frac{Q}{8 C_{2}(t)}+C^{\prime}
$$

Therefore $Q \leq 8 C^{\prime} C_{2}(t) \leq 8 C^{\prime} C_{1}(t)$ at $\left(t_{0}, z_{0}\right)$. By our choice of $C_{1}(t), C_{2}(t)$, $H$ is bounded by some constant $C$ depending only on $\sup \left|\varphi_{0}\right|$ and $\sup \left|\dot{\varphi}_{0}\right|$, which gives the bound for $|\operatorname{Ric}|$.

The estimates we have obtained imply that the parabolic $C^{\alpha, \alpha / 2}$ norm of the coefficients in Equation (3.23) can be bounded. The parabolic Schauder estimates then give a $C^{2+\alpha, 1+\alpha / 2}$ bound for $\dot{\varphi}$ in $\left[\epsilon, T^{\prime}\right] \times \mathrm{M}$ for any $\epsilon>0$ with the bounds only depending on $\epsilon, \sup \left|\varphi_{0}\right|$ and $\sup \left|\dot{\varphi}_{0}\right|$. Similarly we can obtain a $C^{2+\alpha, 1+\alpha / 2}$ bound for $\varphi_{k}, \varphi_{\bar{k}}$ in $\left[\epsilon, T^{\prime}\right] \times M$. Differentiating the flow again and repeatedly using Schauder estimates, we obtain all higher-order estimates for $\varphi$. Let $\epsilon \rightarrow 0$, we obtain the bounds in Proposition 3.1 which blow up as $t \rightarrow 0$. Particularly, there exists a smooth solution on $[0, T]$ where $T$ is the same as in Lemma 3.1 and depends only on $\sup \left|\varphi_{0}\right|$ and $F$. 


\section{Proof of Theorem 1.1}

Assume that $\hat{\omega}$ satisfies the condition (1.3), then it follows from [13] that Kołodziej's stability result (Corollary 4.4 in [11]) is also true. In particular if

$$
\left(\hat{\omega}+\sqrt{-1} \partial \bar{\partial} \phi_{1}\right)^{n}=\left(\hat{\omega}+\sqrt{-1} \partial \bar{\partial} \phi_{2}\right)^{n}=f \hat{\omega}^{n}
$$

with $f \geq 0 \in L^{p}(M, \hat{\omega}), p>1$ and $\int_{M} f \hat{\omega}^{n}=\int_{M} \hat{\omega}^{n}$, then $\phi_{1}-\phi_{2}=$ const. Now suppose that $\phi \in P S H(M, \hat{\omega}) \cap L^{\infty}(M)$ is a weak solution of the equation

$$
(\hat{\omega}+\sqrt{-1} \partial \bar{\partial} \phi)^{n}=\mathrm{e}^{-F(\phi, z)} \hat{\omega}^{n}
$$

then $f(z)=\mathrm{e}^{-F(\phi(z), z)} \in L^{p}(M, \hat{\omega}), p>1$ as $\phi$ is bounded for $t \leq T$. Also, the condition (1.3) gives that $\int_{M} f \hat{\omega}^{n}=\int_{M} \hat{\omega}^{n}$. Therefore, Theorem 5.2 in [3] shows that $\phi$ is continuous. Approximate $\phi$ with a sequence of smooth functions $\phi_{j}$, such that

$$
\sup _{M}\left|\phi_{j}-\phi\right| \rightarrow 0, \text { as } j \rightarrow \infty
$$

It follows from [21] there exist smooth functions $\psi_{j}$, such that

$$
\left(\hat{\omega}+\sqrt{-1} \partial \bar{\partial} \psi_{j}\right)^{n}=c_{j} \mathrm{e}^{-F\left(\phi_{j}, z\right)} \hat{\omega}^{n},
$$

where $c_{j}>0$ are constants chosen to satisfy the integration equality of the above equation. From assumption (1.3) we have $c_{j} \rightarrow 1$ as $j \rightarrow \infty$. Normalize $\psi_{j}$ as in [11]

$$
\sup \left(\psi_{j}-\phi\right)=\sup \left(\phi-\psi_{j}\right)
$$

The stability result from [13] gives

$$
\lim _{j \rightarrow \infty}\left\|\psi_{j}-\phi\right\|_{L^{\infty}}=0
$$

Consider the equations

$$
\frac{\partial \varphi_{j}}{\partial t}=\log \frac{\left(\hat{\omega}+\sqrt{-1} \partial \bar{\partial} \varphi_{j}\right)^{n}}{\hat{\omega}^{n}}+F\left(\varphi_{j}, z\right)-\log c_{j} .
$$

Applying Proposition 3.1, there exist a sequence of smooth functions $\varphi_{j}$ with $\varphi_{j}(0)=\psi_{j}$ such that $\varphi_{j}$ solves the equations on $\left[0, T_{j}\right]$ where $T_{j}$ depends only 
on $\sup \left|\psi_{j}\right|$ and $\sup \left|\dot{\varphi}_{j}(0)\right|$. Using (4.3) and (4.6),

$$
\dot{\varphi}_{j}(0)=F\left(\psi_{j}, z\right)-F\left(\phi_{j}, z\right) .
$$

It follows from (4.2) and (4.5) that $\sup \left|\psi_{j}\right|$ and $\sup \left|\dot{\varphi}_{j}(0)\right|$ can be bounded by a constant depending only on $\sup |\phi|$. Therefore there exists a $T>0$ independent of $j$ such that $\varphi_{j}$ solve the Equation (4.6) on $[0, T]$. By Lemma 6 in [18], $\left\{\varphi_{j}\right\}$ is a Cauchy sequence in $C^{0}([0, T] \times M)$. Let

$$
\beta(t, z)=\lim _{j \rightarrow \infty} \varphi_{j},
$$

which is continuous on $[0, T] \times M$. For any $\epsilon>0$, from the proof of Proposition 3.1, we have bounds on all derivatives of $\varphi_{j}$ for $t \in[\epsilon, T]$. Then $\beta \epsilon$ $C^{\infty}([\epsilon, T] \times M)$ and

$$
\lim _{j \rightarrow \infty}\left\|\beta-\varphi_{j}\right\|_{C^{k}([\epsilon, T] \times M)}=0 .
$$

Lemma 3.1 gives that $\left|\dot{\varphi}_{j}(t)\right| \leq \sup \left|\dot{\varphi}_{j}(0)\right| e^{C t}$, for $t \in[0, T]$. From (4.7) we get

$$
\dot{\varphi}_{j}(0) \rightarrow 0 \text { as } j \rightarrow \infty .
$$

Therefore for any $t>0$,

$$
\dot{\beta}(t)=\lim _{j \rightarrow \infty} \dot{\varphi}_{j}(t)=0 .
$$

As it is continuous on $[0, T]$, we have $\beta(0)=\beta(t)$ for $t \in(0, T]$ is smooth. But $\beta(0)=\lim _{j \rightarrow \infty} \varphi_{j}(0)=\lim _{j \rightarrow \infty} \psi_{j}=\phi$, thus we get the smoothness of $\phi$.

\section{Acknowledgments}

The author thanks her advisor Jiaping Wang for constant support, encouragement and many helpful discussions. The author thanks Valentino Tosatti and Ben Weinkove for suggesting her the problem and helpful comments on the first version of this paper. In addition, I am grateful to Stawomir Kołodziej for the clarification on the stability result and to Haojie Chen for many useful conversations. I also thank the referees for their careful reading of the paper and their comments. 


\section{References}

[1] E. Bedford and B.A. Taylor, The Dirichlet problem for a complex Monge-Ampère equation, Invent. Math. 37(1) (1976), 1-44.

[2] Z. Błocki, A gradient estimate in the Calabi-Yau theorem, Math. Ann. 344(2) (2009), 317-327.

[3] S. Dinew and S. Kołodziej, Pluripotential estimates on compact Hermitian manifolds, arXiv:0910.3937.

[4] P. Gauduchon, Le thèorème de l'excentricite nulle, (French) C. R. Acad. Sci. Paris Sèr. A-B 285(5) (1977), A387-A390.

[5] M. Gill, Convergence of the parabolic complex Monge-Ampère equation on compact Hermitian manifolds, Comm. Anal. Geom. 19(2) (2011), 277-303.

[6] B. Guan and Q. Li, Complex Monge-Ampère equations and totally real submanifolds, Adv. Math. 225(3) (2010), 1185-1223.

[7] A. Hanani, Équations du type de Monge-Ampère sur les variétés Hermitiennes compactes, J. Funct. Anal. 137(1) (1996), 49-75.

[8] M. Klimek, Pluripotential theory, Oxford University Press, New York, 1991.

[9] S. Kołodziej, The complex Monge-Ampère equation, Acta Math. 180(1) (1998), 69-117.

[10] S. Kołodziej, The complex Monge-Ampère equation and pluripotential theory, Mem. Amer. Math. Soc. 178(840) (2005).

[11] S. Kołodziej, The Monge-Ampère equation on compact Kähler manifolds, Indiana Univ. Math. J. 52(3) (2003), 667-686.

[12] S. Kołodziej, Hölder continuity of solutions to the complex MongeAmpère equation with the right-hand side in $L^{p}$ : the case of compact Kähler manifolds, Math. Ann. 342(2) (2008), 379-386.

[13] S. Kołodziej, Email communication. 2013.

[14] D.H. Phong, N. Š $\breve{s u m ~ a n d ~ J . ~ S t u r m, ~ M u l t i p l i e r ~ i d e a l ~ s h e a v e s ~ a n d ~ t h e ~}$ Kähler-Ricci flow, Comm. Anal. Geom. 15(3) (2007), 613-632.

[15] D.H. Phong and J. Sturm, The Dirichlet problem for degenerate complex Monge-Ampère equations, Comm. Anal. Geom. 18(1) (2010), 145-170. 
[16] D.H. Phong, J. Song, J. Sturm and B. Weinkove, On the convergence of the modified Kähler-Ricci flow and solitons, Comment. Math. Helv. 86(1) (2011), 91-112.

[17] J. Song and G. Tian, The Kähler-Ricci flow through singularities, arXiv:0909.4898.

[18] G. Székelyhidi and V. Tosatti, Regularity of weak solutions of a complex Monge-Ampère equation, Anal. PDE 4(3) (2011), 369-378.

[19] M. Sherman and B. Weinkove, Local Calabi and curvature estimates for the Chern-Ricci flow, New York J. Math. 19 (2013), 565-582.

[20] V. Tosatti and B. Weinkove, Estimates for the complex Monge-Ampère equation on Hermitian and balanced manifolds, Asian J. Math. 14(1) (2010), 19-40.

[21] V. Tosatti and B. Weinkove, The complex Monge-Ampère equation on compact Hermitian manifolds, J. Am. Math. Soc. 23(4) (2010), $1187-1195$.

[22] V. Tosatti and B. Weinkove, On the evolution of a Hermitian metric by its Chern-Ricci form, arXiv:1201.0312.

[23] V. Tosatti and B. Weinkove, The Chern-Ricci flow on complex surfaces, Compos. Math. 149(12) (2013), 2101-2138.

[24] V. Tosatti and B. Weinkove, and X. Yang, Collapsing of the ChernRicci flow on elliptic surfaces, arXiv:1302.6545.

[25] S.-T. Yau, On the Ricci curvature of a compact Kähler manifold and the complex Monge-Ampère equation I, Comm. Pure Appl. Math. 31 (1978), 339-411.

School of Mathematics

University OF Minnesota

MinNeAPOLIS, MN 55455

USA

Email address: niexx025@umn.edu

Received November 5, 2013 